\title{
Texture Analysis of Radiometric Signatures of New Sea Ice Forming in Arctic Leads
}

\author{
Duane T. Eppler and L. Dennis Farmer
}

\begin{abstract}
Analysis of 33.6-GHz, high-resolution, passive microwave images suggests that new sea ice accumulating in open leads is characterized by a unique textural signature which can be used to discriminate new ice forming in this environment from adjacent surfaces of similar radiometric temperature. Data analyzed were acquired in March 1988 in conjunction with the joint Navy-NASA SSM/I Sea Ice Validation Experiment using the Navy $\mathrm{Ka}$-band Radiometric Mapping System (KRMS), an airborne passive microwave imager. Ten training areas were selected from the data set, three of which consisted entirely of first-year ice, four entirely of multiyear ice, and three of new ice in open leads in the process of freezing. A simple gradient operator was used to characterize the radiometric texture in each training region in terms of the degree to which radiometric gradients are oriented. New ice in leads has a sufficiently high proportion of well-oriented features to distinguish it uniquely from first-year ice and multiyear ice. The predominance of well-oriented features probably reflects physical processes by which new ice accumulates in open leads. Banded structures, which are evident in aerial photographs of new ice, apparently give rise to the radiometric signature observed here, in which the trend of brightness temperature gradients is aligned parallel to lead trends. First-year ice and multiyear ice, which have been subjected to a more random growth and process history, lack this banded structure and therefore are characterized by signatures in which well-aligned elements are less dominant.
\end{abstract}

Keywords-Passive microwave, sea ice, new ice, leads, ice classification, gradient operator, texture analysis.

\section{INTRODUCTION}

$\mathrm{U}$ NAMBIGUOUS detection of thin, newly formed sea ice in passive microwave images of actively freezing Arctic leads is difficult, because new ice is not characterized by a discrete emissivity or narrow range of brightness temperatures. This is because radiometric characteristics of an actively freezing body of sea water change dramatically as the sea surface changes from liquid to solid.

Passive microwave measurements made using aircraft and surface radiometers show that sea water, which is reflective at microwave wavelengths and has an emissivity of approximately 0.55 , typically is the radiometrically coldest surface observed in passive microwave images of the Arctic pack. Thin, newly formed saline ice, some of which may be thinner than two $\mathrm{cm}$, has an emissivity of approximately 0.95 and typically is the radiometrically warmest surface observed [1], [2]. Laboratory and field measurements show that emissivities and brightness

Manuscript received April 30, 1990; revised August 14, 1990. This work was supported in part by NASA's Snow and Ice Processes Program, in part by the U.S. Navy Satellite Applications Technology (SAT) Program (program element $0603704 \mathrm{~N}$ ), and in part by NOARL under Department of Defense program element $0601153 \mathrm{~N}$ through the Sea Ice Classification Project. This work represents NOARL Contribution No. JA:332:041:90.

The authors are with the Polar Oceanography Branch Office, Naval Oceanographic and Atmospheric Research Laboratory (NOARL), 72 Lyme Road, Hanover, NH 03755-1290.

IEEE Log Number 9041435. temperatures common in freezing leads extend over this entire range. As dispersed ice crystals (frazil) form in the water column, coalesce at the surface in the form of slush and grease ice, and freeze into a consolidated ice sheet, the emissivity (and brightness temperature) of the surface increases progressively from that of open water to that of new ice.

Prior attempts to classify ice types imaged by single-band passive microwave sensors have met with difficulty in cases where new ice was present in actively freezing leads and polynyas [1]. This previous work, which used brightness temperature alone as the basis for classification, produced satisfactory results for scenes in which multiyear ice, first-year ice, and open water were the only surfaces present. In examples where new ice (frazil, slush, grease ice) occurred in conjunction with these other surfaces this method failed, because brightness temperatures typical of multiyear ice and first-year ice fall within the broad range of temperatures typical of new ice.

Here we report a new approach to this classification problem that employs a textural descriptor to discriminate new ice which occurs in freezing leads from other ice types. Our method uses a simple gradient operator to characterize textural aspects of radiometric signatures. In new ice these signatures arise from physical processes by which dispersed ice crystals accumulate to form a continuous ice cover, whereas in multiyear ice and first-year ice they reflect deformational features created after the ice forms a consolidated sheet. Signatures that result are unique with respect to ice type. The technique thus provides a means by which conventional ice-classification methods can be enhanced to extract additional information from passive microwave images concerning the character of sea ice.

\section{Physical Basis for Discrimination of New Ice}

New ice that occurs in the form of frazil, slush, and grease ice in leads and polynyas commonly displays banded structures which are oriented parallel to the lead axis or polynya boundary (Fig. 1). Such bands reflect processes by which dispersed ice crystals (frazil) that form in exposed sea water accumulate. Wind blowing across the lead or polynya exerts stress on the surface layer, cools exposed water, initiates convective mixing, and, if allowed to continue for a sufficient period of time, creates a layer of supercooled water several meters thick at the sea surface [3]. Frazil forms in this supercooled mixed layer, floats to the surface, and is herded to the downwind side of the water opening by wind (Fig. 2). Here it is packed into bands of slush which accumulate against thicker ice at the edge of the lead or polynya [4], [5] (Fig. 1). In the absence of wind, similar structures typified by more loosely packed slush are likely to form. Radiative cooling initiates freezing at the ocean surface. Expulsion of brine from the accumulating frazil mat initiates convection in the upper water column [6]. Convection cells, which 


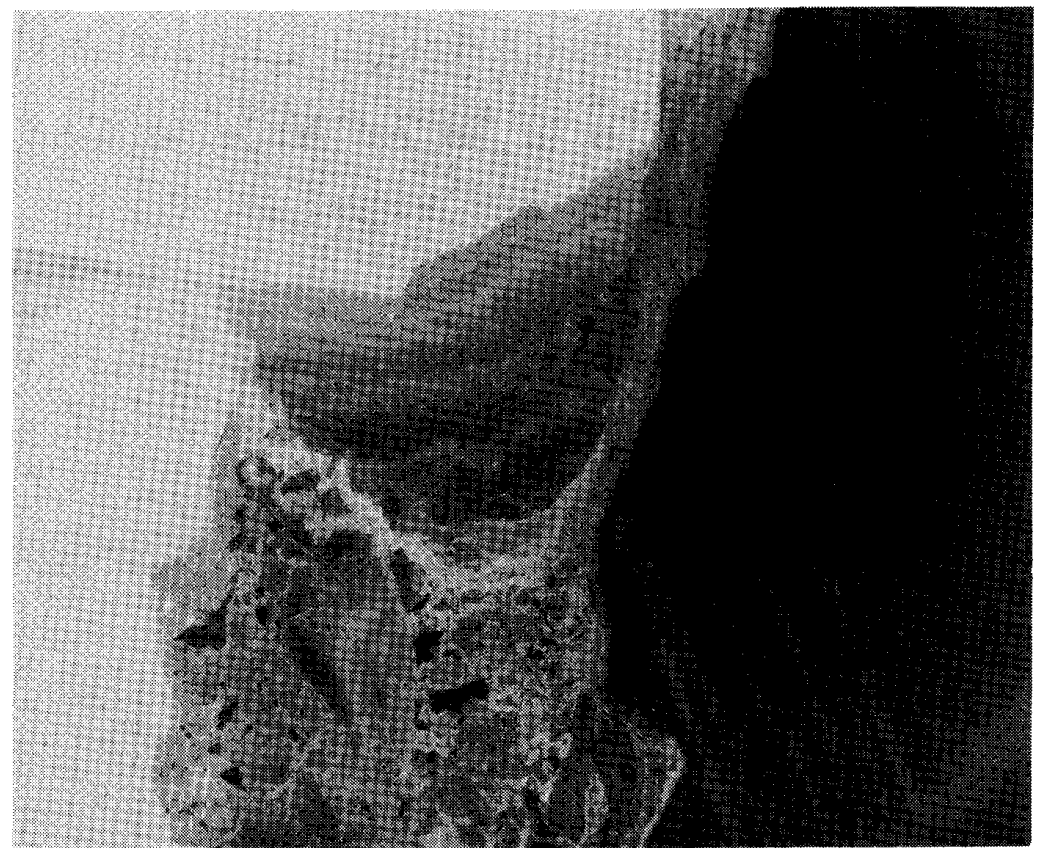

Fig. 1. Aerial photograph showing banded structures in new ice. Wind has packed floating slush along multiyear floes at left and top to form a region of new ice at image center in which banded structures are evident. At the time the photograph was taken, a new band was in the process of forming along the boundary between the water opening and the region of banded new ice. Stringers of slush aligned parallel to wind direction are visible in open water at image right. Ground distance across the image is approximately $1.5 \mathrm{~km}$. Photograph was taken with an RC-10 camera from an altitude of $1525 \mathrm{~m}(5000 \mathrm{ft})$ on March 20,1983 and shows ice conditions at approximately $72^{\circ} \mathrm{N} 157^{\circ} \mathrm{W}$

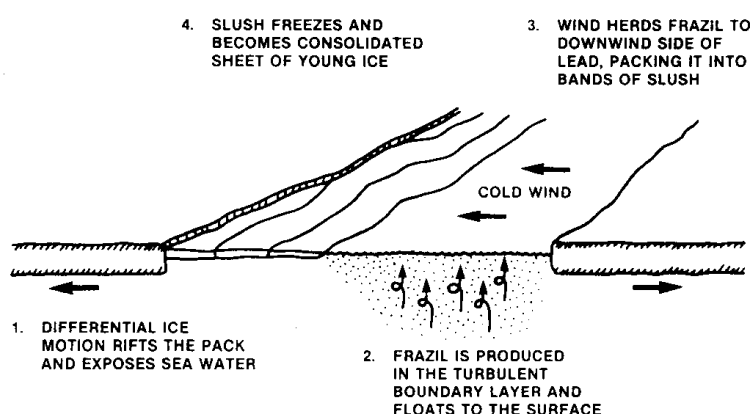

Fig. 2. Conceptual model of formation of banded structures in thin ice occurring in leads based on work by Pease [5]. Small-scale movement of adjacent ice sheets may impose secondary sets of linear features on the consolidating ice sheet in the form of folds, rafts, rifts, and wrinkles.

are aligned parallel to the lead trend, herd frazil into bands which extend along the length of the lead.

The volume of frazil generated in a given period of time depends on heat flux from ocean to atmosphere, which is determined by wind speed, air temperature, and long- and shortwave radiative fluxes [4]. Natural temporal variability of these factors modulates both the flux of frazil and the rate of accumulation of slush at the downwind edge of the lead. Assuming that air temperature and long- and shortwave radiation either remain constant or vary slowly over extended time periods, wind speed is the dominant variable contributing to short-term (hour-to- hour) variation in the frazil flux and accumulation rate. Large volumes of slush should accumulate during large stress events (periods of elevated wind speed). During intervening periods of lower stress frazil flux will decline, and the newly deposited slush band will begin to drain and then consolidate as adjacent crystals freeze together. The banded structure typical of new ice in leads, then, probably arises in large part from repeated episodes of high and low wind stress.

Adjacent bands are distinguished in photographs by differences in grey tone (albedo) (Fig. 1). The tone displayed by a particular band probably is a function of the liquid water content of the slush. We speculate that light tones observed in photographs correspond to low liquid water content, and that dark tones correspond to high liquid water content. It follows from this hypothesis that light-toned bands either (i) are elevated above sea level (perhaps due to thicker accumulation of slush at that site) and have drained, (ii) are compacted and have low porosity, or (iii) have become consolidated by the freezing of entrapped water. Conversely, dark-toned bands are likely to be flooded, porous, or largely unconsolidated, although this remains to be documented by surface measurements.

These banded structures also are evident in passive microwave images of new ice (Fig. 3). Large-scale features that appear in photographs commonly are well defined in the microwave images, although many small-scale details are portrayed less clearly. This is a function of differences both in the resolving power of the two sensors (centimeters in cameras, tens of meters in imaging microwave radiometers), and in electromagnetic properties of the surface at frequencies sensed by each 


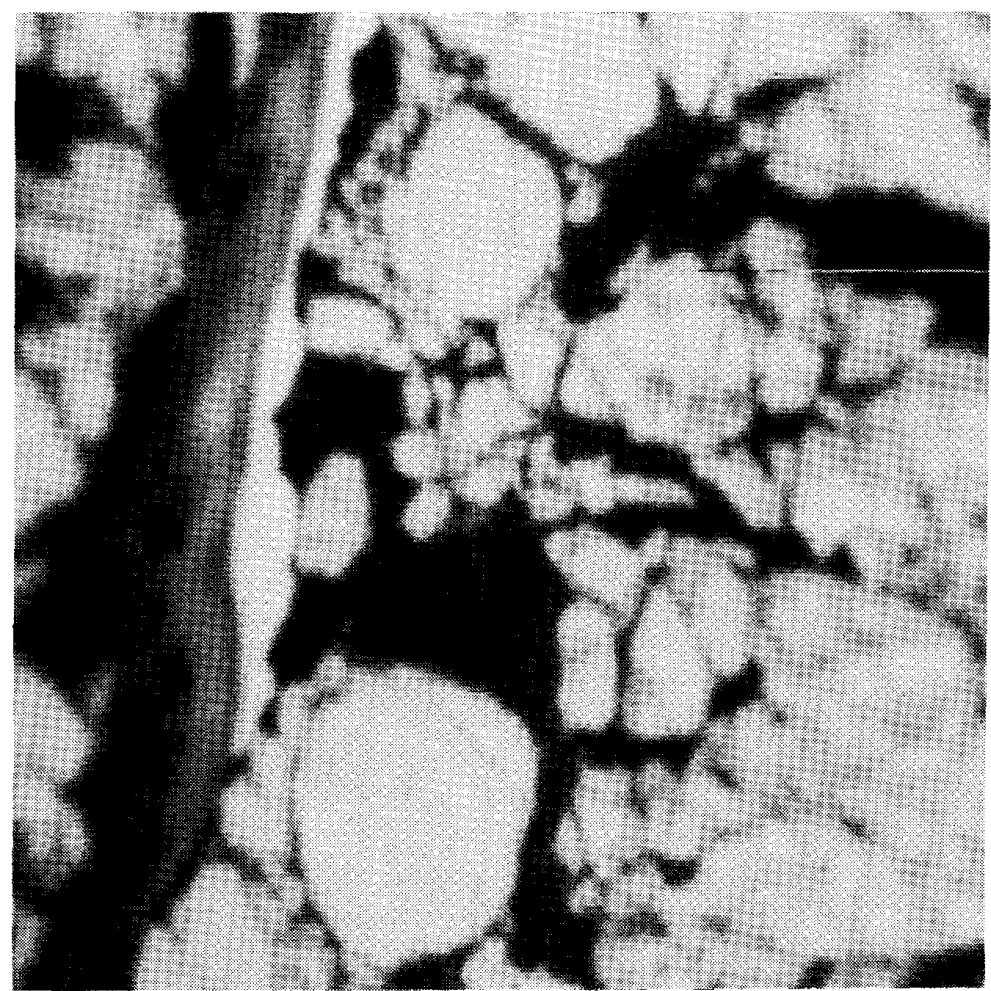

Fig. 3. KRMS passive microwave image of sea ice. Radiometrically cool floes of old ice are present in the center and righthand portions of the image (light grey areas). These floes are surrounded by a matrix of radiometrically warm first-year ice (dark toned areas). A lead extends from top to bottom at the left-hand side of the image. Open water present along the right-hand side of the lead is evident as a radiometrically cold, narrow, white band extending about three-quarters of the way down the image. New ice that has formed recently in the lead occurs to the left of the open water, as evidenced by a series of grey bands aligned parallel to the lead trend. Ground distance across the scene is approximately $14.5 \mathrm{~km}$. The image was acquired on March 11 , 1988 at approximately $79^{\circ} \mathrm{N} 128^{\circ} \mathrm{W}$ from an altitude of $3050 \mathrm{~m}(10000 \mathrm{ft})$.

instrument. Surface conditions that give rise to significant differences in albedo that are sensed by the camera may produce lower contrast differences in emissivity at the frequency sensed by the microwave radiometer, and vice versa.

Grey tones in passive microwave images (Fig. 3) correspond to differences in the radiometric brightness temperature, which is a function of surface emissivity. Field and laboratory experiments show that the brightness temperature of a sheet of new ice freezing under quiescent conditions rises from radiometrically cool temperature when the scene consists entirely of open water, to warm temperatures near the ambient physical temperature when a thin continuous skin of ice covers the surface [1], [2]. As long as an unconsolidated ice sheet exists, the surface consists of a mixture of solid ice crystals dispersed in water. The radiometric temperature of the scene during this time is a function, in large part, of the relative proportion of radiometrically cool liquid and radiometrically warm solid phases which are present.

Bands of slush respond similarly in a radiometric sense. Porous bands that contain high percentages of liquid water at the surface will be radiometrically cooler than compact bands or bands that have consolidated and become dry through freezing. Bands that are topographically high and drained also may appear radiometrically warm, although enhanced thermal cooling of these features induced by their protrusion into the boundary layer probably reduces somewhat their radiometric contrast with adjacent porous bands. On the other hand, this same thermal cooling is likely to enhance their contrast with adjacent radiometrically warm, low relief, compact, or frozen bands, thereby contributing to the overall banded signature of new ice.

As the slush freezes into a consolidated ice sheet, gentle movement of ice bounding the lead may deform the new ice further and create additional sets of oriented features in the form of rifts, rafts, ridges, and folds. Rifts and tears in the new ice sheet, it appears from an analysis of photographs, typically are oriented perpendicular or subperpendicular to the lead trend, particularly when the motion of the ice on opposing sides of the lead includes shear. Rafts, which arise from compression, also include structural elements oriented perpendicular to the lead trend. Rifts especially will produce radiometric signatures, because they expose water. This adds a secondary set of linear radiometric features, orthogonal in trend to the primary banded structures formed by accumulating slush, to new ice characteristics.

The linear fabric imparted first by wind-driven processes to new ice accumulating in leads, and then by subsequent deformation, should thus be present in the microwave data. This fabric will be expressed as sets of well-aligned features defined: 
(a) By boundaries between slush bands of different physical and radiometric character; and (b) by orthogonal deformational features within these bands. These patterns will persist in the newly formed ice sheet only until consolidation is complete and the surface has frozen uniformly. Beyond this point the ice surface will be uniformly emissive and will lack the radiometric contrast required to distinguish individual bands. The linear fabric thus marks a particular stage in the development of pack ice that indicates a unique environment which is of geophysical interest.

Neither first-year ice nor multiyear ice contain linear features which produce radiometric signatures which are aligned as well as those typical of new ice accumulating in leads. In contrast to new ice, first-year ice contains few linear features which leave signatures in passive microwave images. Ridges and fractures are composed of saline ice with radiometric properties similar to adjacent first-year ice and therefore are characterized at best by discontinuous low-contrast signatures. Analysis of ridge orientations by Mock et al. [7] suggests that these features, even if they do leave signatures which can be detected, are not likely to be strongly aligned. Although linear features are more common in multiyear ice (ridges, hummocks, fractures, meltpond boundaries, drifted snow), most of these features arise from a range of independent processes operating sporadically over long periods of time. During intervals between successive events, floes drift and rotate and stress fields change orientation. Signatures related to the collection of linear features observed across multiyear floes thus will be characterized by random orientations rather than by strong alignment.

This, then, provides a model for discriminating new ice from other surfaces of similar radiometric temperature in passive microwave images: New ice will display textures which are characterized by well-aligned sets of linear features; multiyear ice and first-year ice will display textures which are characterized by fewer linear features which are poorly or randomly aligned.

\section{Method of ANALysis}

Passive microwave images which show new ice freezing in open leads were selected for analysis from data obtained in conjunction with the NASA-Navy SSM/I validation data set in March 1988 [8]. These data were acquired along a flight track which extends from Harrison Bay, Alaska, to Ellesmere Island with the Navy $K a$-band Radiometric Mapping System (KRMS), an airborne passive microwave imager which senses vertically polarized radiation at a center frequency of $33.6 \mathrm{GHz}$. Detailed descriptions of the instrument and methods used to process KRMS data are provided in [1], [9]-[13]. Images used for this study are 512 pixels wide and show a swath approximately 14.5 $\mathrm{km}$ across. Each pixel covers an area $28.3 \times 28.3 \mathrm{~m}$ on the ground.

\section{A. Differential Gradient Operator}

The KRMS data set included examples of three open leads in the process of freezing. Training areas representative of new ice, first-year ice, and multiyear ice were identified in these images. A total of three areas were selected to represent new ice, three to represent first-year ice, and four to represent multiyear ice. A simple differential gradient operator based on the work of Roberts [14] and Kirsch [15] was applied to the images. The gradient operator consists of two $3 \times 3$ pixel masks (Fig. 4). The masks are weighted to measure orthogonal components of the gradient within the $3 \times 3$ neighborhood surrounding the

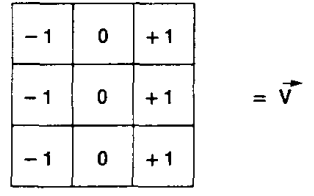

(a)

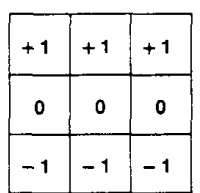

$=\overrightarrow{\mathrm{H}}$

(b)

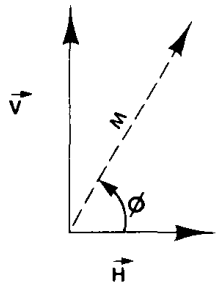

(c)

Fig. 4. Gradient operator based on the work of Roberts [14] and Kirsh [15] that was applied to images analyzed in this study. (a) Weighted mask used to compute the vertical component of gradient. (b) Weighted mask used to compute the horizontal component of gradient. (c) Geometric relationship between the vertical and horizontal gradient vectors, and the magnitude and orientation of the gradient.

center pixel. Weights in one mask measure the vertical component of the gradient (vertical being the direction parallel to pixel columns) (Fig. 4(a)); weights in the other mask measure the horizontal component (parallel to pixel rows) (Fig. 4(b)). The operator is implemented by computing for each pixel the sum of the products between the weights and the pixel intensities for each mask:

$$
\vec{V}=\sum_{r=1}^{3} \sum_{c=1}^{3}\left(w v_{r, c} * i_{r, c}\right)
$$

and

$$
\vec{H}=\sum_{r=1}^{3} \sum_{c=1}^{3}\left(w h_{r, c} * i_{r, c}\right)
$$

where $\vec{V}$ and $\vec{H}$ are the vertical and horizontal components of the gradient at the pixel in the center of the mask, $r$ and $c$ are row and column indices within the $3 \times 3$ neighborhood, $w v$ and wh are weights in the vertical and horizontal masks (Fig. 4(a) and 4(b)), and $i$ is the intensity of the pixel in the raw image.

The vertical and horizontal components of the gradient in effect are vectors, the lengths of which are measures of the magnitude of the gradient in the vertical and horizontal directions. Adding these two orthogonal vectors yields a third vector which represents the true magnitude $(M)$ and orientation $(\phi)$ of the gradient (Fig. 4(c)):

$$
M=\sqrt{\vec{V}^{2}+\vec{H}^{2}}
$$

and

$$
\phi=\tan ^{-1}(\vec{V} / \vec{H}) .
$$




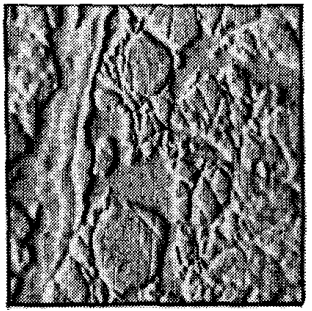

$\vec{v}$

(a)

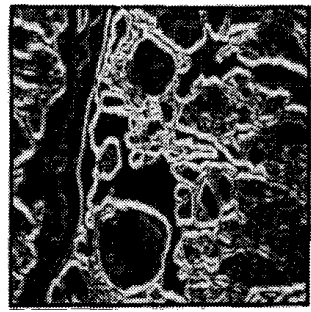

M

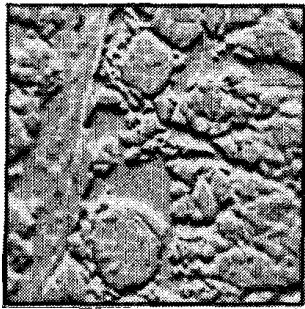

$\vec{H}$

(b)

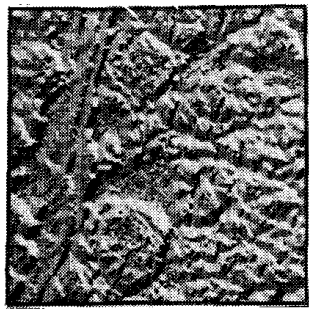

$\varnothing$

(d)

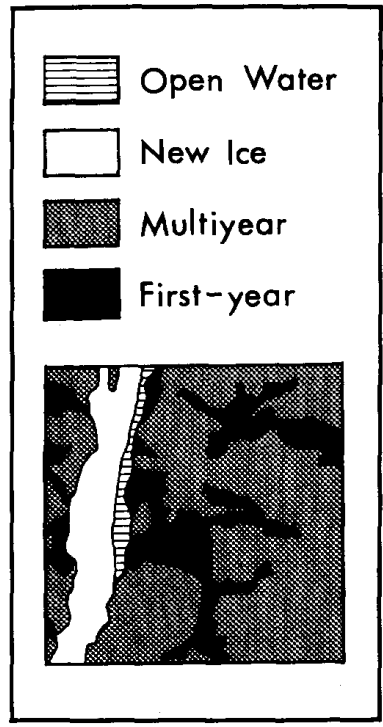

INTERPRETATION

(e)

Fig. 5. Result of applying the gradient operator shown in Fig. 4 to the passive microwave image shown in Fig. 3. Grey level represents the relative magnitude of each variable; dark tones correspond to low values, and light tones to high values. (a) Vertical gradient component; enhances features trending from top to bottom in the image. (b) Horizontal gradient component; enhances features trending across the image. (c) Gradient magnitude; marks boundaries between features of different brightness temperature and enhances edges. (d) Gradient orientation; shows trend of gradients. (e) Map of surfaces present in the scene, derived manually by interpreting the image of radiances shown in Fig. 3.

Images containing the training areas were processed using this operator. The results for each scene were displayed as a series of four images, one each for the vertical and horizontal vector magnitudes, and one each for the gradient magnitude and orientation. These images were created by mapping values for $\vec{V}, \vec{H}, M$, and $\phi$ as pixel intensities at respective row and column image coordinates. Fig. 5 shows the result of applying the gradient operator to the scene shown in Fig. 3. Dark tones correspond to low values, and light tones to high values in Fig. 5(a) through (c). In Fig. 5(d), $\phi$ varies from $0^{\circ}$ (black; gradient oriented vertically with high radiance surface to left of gradient) clockwise to $180^{\circ}$ (middle grey; gradient oriented vertically with high radiance surface to right of gradient) clockwise to $360^{\circ}$ (white; gradient orientation essentially the same as $0^{\circ}$ ). Fig. 5(e) shows a map of surface types in the scene interpreted manually from Fig. 3 . Note that $\vec{V}$ accentuates features that trend vertically (Fig. 5(a)), and $\vec{H}$ accentuates features that trend horizontally (Fig. 5(b)). Boundaries between different ice types are the sites of both greatest magnitude in gradients (Fig. 5(c)) and strongest alignment in the trend of gradients (Fig. 5(d)).

Visual examination of patterns and textures observed in images of $\vec{V}, \vec{H}, M$, and $\phi$ indicate that areas of first-year ice, multiyear ice, and new ice in leads are characterized by different signatures. This is especially true for $\phi$ (Fig. 5(d)). Local variability in $\phi$ appears to be highest for first-year ice, as suggested by the speckled pattern caused by the absence of welloriented gradients in adjacent pixels. Some areas of multiyear floes show similar speckled patterns, but the overall multiyear texture differs from the first-year texture in that the multiyear regions of unaligned features are separated by areas in which adjacent pixels show well-aligned gradients (areas of relatively consistent grey tone) which presumably correspond to ridges and fractures, and probably in part to meltponds as well.

New ice signatures differ from those of both first-year ice and multiyear ice in that new ice shows both greater local homogeneity, especially for $\phi$, and evidence of broader scale trends. For example, visual analysis of $\phi$ images (Fig. 5(d)) suggests that linear features marked by gradients (apparent in Fig. 5(a) and (c)) show moderate to strong alignment in directions either generally parallel to lead orientations (black or white features) or generally perpendicular to lead orientations (uniform to mottled grey areas) in regions of new ice. On a pixel-to-pixel scale, individual gradients probably reflect radiometric characteristics of the surface that arise from the underlying fine-scale banded structure of new ice (Fig. 1), or from cross-band rifts and rafts. On a broader scale, general patterns of variation shown by gradients in new ice (Fig. 5(d)) correspond to the larger scale banded structures evident in Figs. 1 and 3 . In contrast, features in first-year ice are unaligned and do not define broad-scale patterns. Features in multiyear ice, although they show local alignment over particular features on the floe, do not show consistent alignment from feature to feature across the floe.

These observations are consistent with the conceptual model presented above. Analysis of the alignment of radiometric gradients thus seems to provide a basis for discriminating new ice from other ice types, at least in a qualitative sense. Next we develop a numeric method of comparison that is sensitive to observed textures and patterns of variation in $\phi$.

\section{B. Circular Variance of $\phi$}

Variability of $\phi$ as shown in Fig. 5(d) suggests that local variance in the alignment of gradients may provide sufficient infor- 


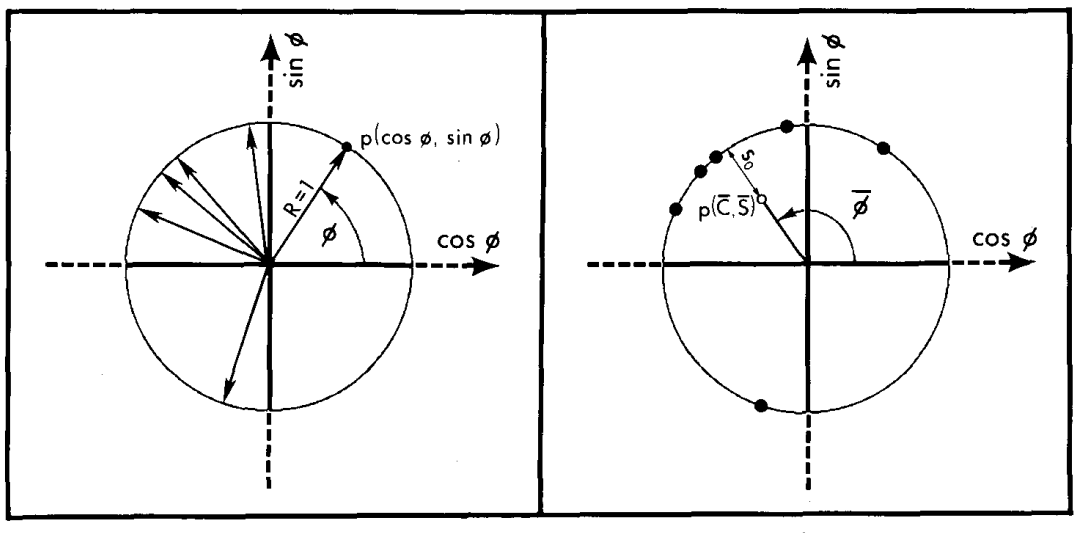

(a)

(b)

Fig. 6. Geometric relationship between a population of orientations $(\phi)$ and their circular mean $(\bar{\phi})$ and variance $\left(s_{0}\right)$. (a) Each angle $\phi$ is represented as a point on a unit circle. The coordinates of these points are the cosines and sines of the angles. (b) The coordinate of the center of gravity of these points (open circle) is the mean cosine $(\bar{C})$ and the mean sine $(\bar{S})$ of the angles. Mardia [16] defines the circular mean $\bar{\phi}$ as the angle between the abscissa and the center of gravity, and the circular variance $s_{0}$ as the distance between the center of gravity and the unit circle.

mation to discriminate new ice uniquely. Inasmuch as $\phi$ is an angular measure, methods presented by Mardia [16] to compute statistics from circular frequency distributions are applied to $\phi$.

Angular measurements are plotted as a set of unit vectors with coordinates $(R, \phi)$, where $R$ is 1 and $\phi$ is the angular distance between the unit vector and a common reference point (Fig. 6). These vectors define a set of points which fall on a unit circle centered at the origin of the cartesian coordinate system described by $(\cos (\phi), \sin (\phi))$. Mardia [16] defines the circular mean and variance in terms of a vector to the center of gravity of these points. The center of gravity, which is the mean vector $(\bar{R}, \bar{\phi})$ of the set of $(R, \phi)$ vectors, is defined in $(\cos (\phi), \sin$ $(\phi))$ space in terms of the mean of the $\cos (\phi)$ and $\sin (\phi)$ coordinates $\bar{C}$ and $\bar{S}$ of the set of points on the unit circle:

$$
\bar{C}=\frac{1}{n} \sum_{i=1}^{n} \cos \phi_{i}
$$

and

$$
\bar{S}=\frac{1}{n} \sum_{i=1}^{n} \sin \phi_{i}
$$

The orientation of this vector as expressed in $(R, \phi)$ coordinates is the circular mean $(\bar{\phi})$, and the circular variance $\left(s_{0}\right)$ is the coverse of its length:

$$
\bar{\phi}=\tan ^{-1}\left(\frac{\bar{S}}{\bar{C}}\right)
$$

and

$$
s_{0}=1-\sqrt{(\bar{C})^{2}+(\bar{S})^{2}} .
$$

Values of $s_{0}$ fall between 0.0 and 1.0. Low variances correspond to unimodal circular distributions that show strong orjentation in a single direction. High variances correspond to polymodal distributions or uniform distributions (Fig. 7). An interesting characteristic of circular variance is that the $s_{0}$ statistic is a maximum for highly symmetric distributions. It can be shown that the center of gravity of any perfectly symmetric

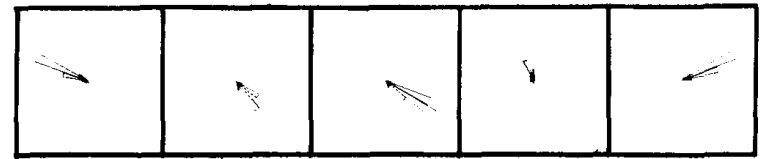

(a)

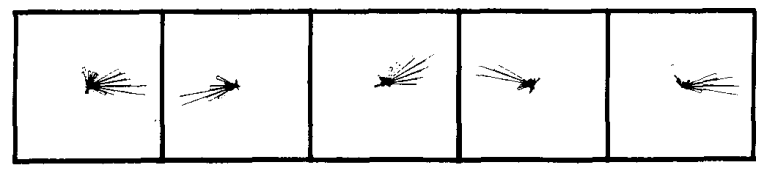

(b)

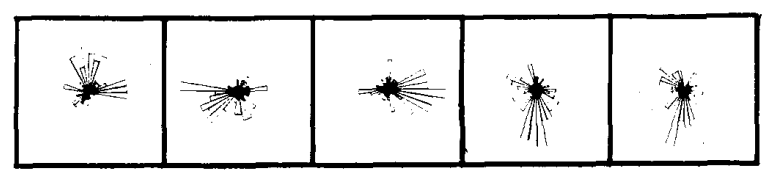

(c)

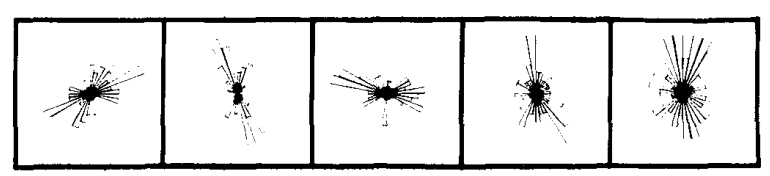

(d)

Fig. 7. The relationship between the circular variance $\left(s_{0}\right)$ and the frequency distribution of angles. Circular variances for histograms in each row fall within the range of values shown to the left. Low variances correspond to distributions that are unimodal and show strong orientation in a single direction. As the variance increases, the distributions become polymodal. Data shown were selected randomly from training regions used in this study. (a) 0 to 0.1 . (b) 0.3 to 0.4 . (c) 0.6 to 0.7 . (d) 0.9 to 1.0 .

nonunimodal distribution coincides with the origin or the coordinate system. When perfect symmetry exists, every unit vector $\left(R_{n}, \phi_{n}\right)$ (Fig. 6(a)) is paired with a companion vector $(R$, $\left.\phi_{n+} \pi\right)$ which plots on the opposite side of the circle. Because the two vectors are located exactly $180^{\circ}$ apart and are of the same length, the mean vector of every pair has zero length and 
thus is a point located at the center of the circle. If perfect symmetry exists, all vectors in the distribution are paired, in which case $\bar{\phi}$ is undefined and $(1-\bar{R})$ is 1 . In some sense, then, $s_{0}$ is a relative measure of symmetry in angular data.

\section{Results}

The local circular variance of $\phi$ was computed for images containing training regions for new ice, first-year ice, and multiyear ice. Each image was divided into grid cells 10 by 10 pixels $(283 \times 283 \mathrm{~m})$ in size; $s_{0}$ was computed for the 100 measurements of $\phi$ in each cell. Fig. 8 shows histograms of variances computed for training areas for each surface. Data from different training areas are pooled for each ice type. Histogram intervals were set using the maximum entropy method of Full et al. [17], which provides for optimum discrimination between samples when frequency distributions are compared. Histogram intervals to the left of center (values of $s_{0}$ less than 0.56 ) represent $10 \times 10$-pixel grid cells in the image in which gradients are moderately well aligned to strongly aligned in a single direction. Intervals to the right of center represent cells in which gradients fail to show strong alignment.

Each ice type is characterized by a different frequency distribution of variances (Fig. 8). The distribution for new ice, which is skewed toward high variances, contains the largest population of well-aligned gradients, as the mode on the left of the histogram indicates. This reflects the linear fabric of new ice described previously. The distribution for first-year ice, which is skewed toward low variances, contains the smallest population of well-aligned gradients. This reflects the general absence of coherent linear features which leave signatures consisting of well-aligned elements in passive microwave images of first-year ice. The distribution for multiyear ice is essentially uniform and shows approximately equal populations of cells with wellaligned and poorly aligned gradients. This reflects the mix of deformed ice (fractures, ridges) and undeformed ice (meltponds, interridge regions) that is typical of multiyear floes.

Each of the three ice types can be discriminated numerically on the basis of the percentage of low variance cells in the distribution (cells with strongly oriented gradients). The sum of frequencies in intervals to the left of the midpoint of the distribution ( 0.56 in the case of histograms in Fig. 8) provides a statistic that describes each ice type uniquely. Fig. 9(a) shows the percent of cells in this leftmost half of the distribution for each of the three ice types. Each ice type falls within a discrete range of values. Note that this is true not only for the pooled training samples (large dots), but for the individual training regions as well (small dots). For example, in the case of first-year ice, values range from approximately 20 to $30 \%$, in the case of multiyear ice from approximately 35 to $50 \%$, and in the case of new ice from approximately 55 to $80 \%$.

This analysis includes orientation data from all pixels in the training region regardless of the magnitude of the gradient. For pixels at which the magnitude approaches zero the orientation is of questionable meaning, since the gradient at that point in the image is poorly defined. In an attempt to improve discrimination between the three ice types, orientations which correspond to pixels with low magnitude gradients ( $M$, equation (3), Fig. 5(c)) were eliminated from consideration. The percentage of each distribution consisting of strongly aligned gradients was recomputed using only those orientations associated with gradient magnitudes $>5.0,>10.0,>15.0,>20.0$, and $>25.0$ (Fig. 9(b)). Surprisingly, the best separation between the three
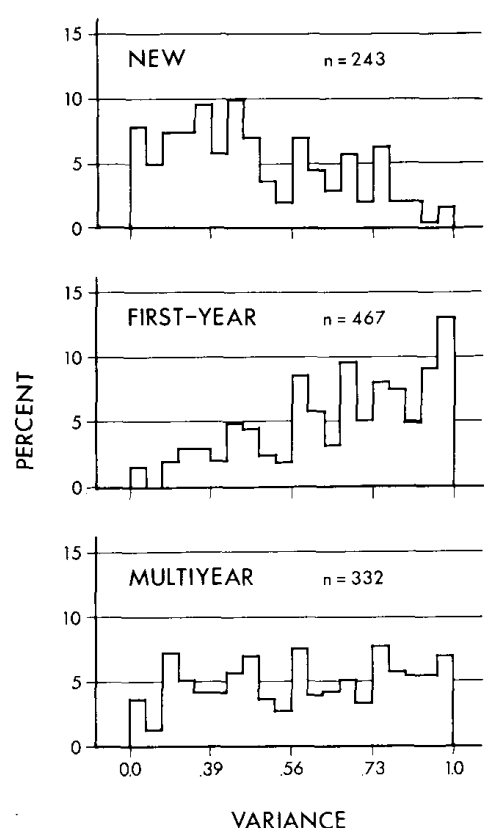

Fig. 8. Frequency distributions of the circular variance of gradient orientations that occur within training areas for new ice, first-year, and multiyear ice. Data are pooled for all training regions sampled for each ice type. Each observation represents data from 100 pixels in the image. Data shown for new ice, for example, account for variance in 24300 pixels. Each ice type displays a unique frequency distribution. Interval boundaries determined using the maximum entropy method of Full et al. [17].

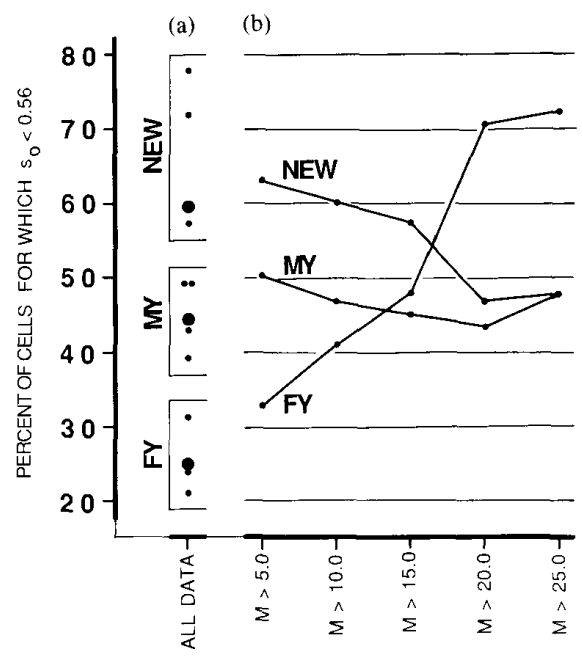

Fig. 9. Discrimination between passive microwave signatures typical of different ice types on the basis of the relative abundance of well-aligned gradients. The statistics used to discriminate between ice types is the percent of the distribution that falls in the left-half of histograms shown in Fig. 8 (i.e., $s_{0}<0.56$ ). (a) Small dots represent distributions for each of the training areas; large dots represent pooled data from all training areas for each ice type and correspond to histograms in Fig. 8. Data for each ice type occupy a discrete range, indicating that the statistic used here effectively discriminates new ice, first-year ice, and multiyear ice signatures. (b) This same statistic plotted for representative samples of the pooled data (large dots) from which orientations associated with low-magnitude gradients have been eliminated. Elimination of these weak features does not improve discrimination, and in most cases makes it worse. 
ice types occurs when all the data are considered (Fig. 9(a)). Eliminating low magnitude gradients increases the likelihood of confusion between two or more ice types for all but the $>5.0$ class, as shown by Fig. 9(b). This result indicates that low magnitude gradients carry significant information which is required to characterize the oriented fabric typical of consolidating sheets of new ice.

\section{SUMmary AND DiscusSION}

Our results suggest that radiometric gradients observed across sea ice imaged by high-resolution passive microwave sensors can be used to discriminate between new sea ice forming in leads, first-year sea ice, and multiyear sea ice. Orientation data derived from a simple gradient operator [14], [15] that was applied to KRMS images contain sufficient information to uniquely characterize radiometric textures typical of these three surfaces in training images that were analyzed.

Banded structures that occur in new ice which is present in actively freezing leads (Fig. 1) create a radiometric texture which is composed of orthogonal linear elements strongly oriented parallel to and perpendicular to the lead trend. These bands form when dispersed frazil crystallizes throughout the mixed layer after seawater is exposed to frigid air. Wind and circulation cells within the lead herd frazil crystals which rise to the water surface into bands of slush, which then accumulate along the downwind side of the lead parallel to the lead trend (Fig. 2). Subsequent deformation of consolidating slush bands by shear may create rifts oriented perpendicular to the lead trend. These processes impart a characteristic radiometric texture of well-aligned linear features to the growing ice sheet. This fabric persists until consolidation is complete.

Comparable sets of strongly oriented gradients are not observed in training images of either first-year ice or multiyear ice. First-year ice exhibits the lowest proportion of well-aligned radiometric gradients; multiyear ice exhibits approximately equal proportions of well-aligned and poorly aligned gradients (Fig. 8). First-year ice generally lacks linear features which leave strong radiometric signatures, a fact that the textural signature reflects. Multiyear floes commonly include a diverse assortment of meltponds, ridges, hummocks, and fractures, which each have distinct radiometric signatures. Although each of these features typically contains strong linear elements, elements from one feature are unlikely to be well aligned with respect to those of another. The resultant multiyear signature thus includes more well-aligned gradients than that of first-year ice, but fewer than the signature of new ice.

Such differences, which are confirmed visually in images that have been filtered using the gradient operator (Fig. 5), can be quantified using the circular variance of gradient orientations (equation (8), Fig. 6). Histograms of the frequency distributions of circular variances measured for different ice types show that each distribution displays a unique shape: The distribution of variances for new ice is skewed right; for multiyear ice it is uniform; and for first-year ice it is skewed left (Fig. 8). The percentage of frequency counts in the left tail of these distributions provides a statistic which can be used to discriminate new ice from first-year ice and multiyear ice (Fig. 9).

This method for discriminating new lead ice from other ice types has been developed using images from a single data set. It remains to be demonstrated that the technique can be applied with equal success to new lead ice which occurs in other Arctic regions during other seasons. The genetic model presented above for accumulation and consolidation of new ice in freezing leads describes the dominant mechanism by which leads freeze and new ice forms [3]-[6]. A key feature of this process is the incorporation of slush bands into the consolidating ice sheet. These bands, which give rise to unique radiometric signatures, are characteristic features of new ice formed in freezing leads across the Arctic, not only in examples imaged in data analyzed here. Most occurrences of new lead ice formed under these conditions thus are expected to show similar signatures, and there is good reason to expect that the method will be effective when applied to data from other regions.

As noted above, the chief variable affecting the character of the bands probably is wind stress. High velocity winds produce well-compacted slush bands; gentle winds produce loose bands. We speculate that the number of bands in a given lead, the width of each band, and the radiometric contrast which results from the relative degree of compactness between adjacent bands is a function of wind. Radiometric gradients measured for new ice in other leads thus may show higher or lower magnitudes $(M$, equation (3)) than new ice in training images used here and the width of individual bands may be narrower or wider, but the fundamental nature of the signatures will remain the same. Analysis of our ability to discriminate new ice as a function of gradient magnitude indicates that the method is robust for low magnitude gradients (Fig. 9). This suggests that the method will be effective even when the contrast between bands is low.

This technique, when used in conjunction with classical sea ice classification algorithms which capitalize only on pixel radiance [1], should produce more consistent and more reliable classifications of high-resolution passive microwave images that contain open water, new ice, first-year ice, and multiyear ice than are now possible. The pilot study reported here demonstrates the use of the technique on a relatively small number of images from a single data set. Additional work is required to determine the extent to which the technique is applicable to data acquired with other passive microwave sensors operating with different spatial resolutions in other regions. It is important to note, however, that physical processes which created radiometric signatures observed in images analyzed here are typical of those operative in freezing leads across the high Arctic. In this light the technique holds promise as a general tool which can be applied to other data to detect and characterize this important Arctic environment.

\section{ACKNOWLEDGMENT}

Critical reviews by F. M. Fetterer, A. W. Green, W. B. Tucker III, and three anonymous reviewers, and discussions with A. W. Lohanick improved the manuscript significantly.

\section{REFERENCES}

[1] D. T. Eppler, L. D. Farmer, A. L. Lohanick, and M. C. Hoover, "Classification of sea ice types with single-band $(33.6 \mathrm{GHz})$ airborne passive microwave imagery," J. Geophys. Res., vol. 91, no. C9, pp. $10661-10695,1986$.

[2] T. C. Grenfell, D. L. Bell, A. L. Lohanick, C. T. Swift, and K. St. Germain, "Multifrequency passive microwave observations of saline ice grown in a tank," in Proc. IGARSS'88 (Edinburgh, UK), 1988, pp. 1687-1690.

[3] W. F. Weeks and S. F. Ackley, "The growth, structure, and properties of sea ice,"' CRREL, Mono. No. 82-1, 1982, 130 pp. [4] J. Bauer and S. Martin, "A model of grease ice growth in small leads," J. Geophys. Res., vol. 88, no. C5, pp. 2917-2925, 1983.

[5] C. H. Pease, "The size of wind-driven coastal polynyas," $J$. Geophys. Res., vol. 92, no. C7, pp. 7049-7059, 1987. 
[6] T. L. Kozo, "Initial model results for Arctic mixed layer circulation under a refreezing lead," $J$. Geophys. Res., vol. 88, no. C5, pp. 2926-2934, 1983.

[7] S. J. Mock, A. Hartwell, and W. D. Hibler, "Spatial aspects of pressure ridge statistics," J. Geophys. Res., vol. 77, no. 30, pp. 5945-5953, 1972.

[8] D. J. Cavalieri et al., "NASA DMSP SSM/I sea ice validation program," NASA, Washington, DC, Tech. Memo., 1990.

[9] B. M. Heydlauff and J. B. Seybold, "Cross-track correction circuit (Navy Technical Catalog No. 1723; Navy Case No. 66637),' Navy Tech. Disclosure Bull., vol. 9, no. 2, 1983, pp. 43-51.

[10] D. T. Eppler, L. D. Farmer, A. L. Lohanick, and M. C. Hoover, "Digital analysis of passive $\mathrm{Ka}$-band microwave images for sea ice classification," NORDA, Stennis Space Center, MS, Tech. Rep. 51, 1984, 54 pp.

[11] L. D. Farmer, D. T. Eppler, and A. L. Lohanick, “Converting digital KRMS values to Kelvin units of brightness temperature,' NORDA, Stennis Space Center, MS, Tech. Note 427, 1990, 16 pp.

[12] L. D. Farmer, D. T. Eppler, and A. L. Lohanick, "Converting digital passive microwave radiances to Kelvin units of brightness temperature,' in Proc. IGARSS'89 (Vancouver, BC), 1989, vol. 1 , pp. 295-296.

[13] D. T. Eppler and B. M. Heydlauff, "Digitizing KRMS analog data on a personal computer," NORDA, Stennis Space Center, MS, Tech. Rep. 219, 1990.

[14] L. G. Roberts, "Machine perception of three-dimensional solids," in Optical and Electro-Optical Information Processing, J. Tippet, Ed. Cambridge MA: MIT Press, 1965, pp. 159-197.

[15] R. A. Kirsch, "Computer determination of the constituent structure of biological images," Computers and Biomedical Res., vol. 4. no. 3, pp. 315-328, 1971

[16] K. V. Mardia, Statistics of Directional Data. New York: Academic, 1972.

[17] W. E. Full, R. Ehrlich, and S. K. Kennedy, "Optimal configuration and information content of sets of frequency distributions," J. Sed. Petrol., vol. 54, no. 1, pp. 117-126, 1984.

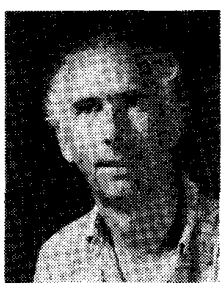

Duane T. Eppler was born in Summit, NJ, on August 12, 1949. He received the B.S. degree with highest honors from St. Lawrence University in 1971, the M.S. degree from Syracuse University in 1973, and the Ph.D. degree from the University of South Carolina in 1980 , all in geology

Since 1981 he has worked with the Polar Oceanography Branch of the Naval Oceanographic and Atmospheric Research Laboratory (NOARL), Hanover, NH. His current research involves analysis of aircraft and satellite passive microwave imagery for purposes of validation and verification of operational products, scene classification, and derivation of environmental sea-ice information

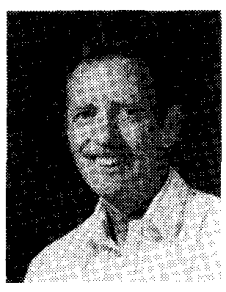

L. Dennis Farmer was born in Portland, ME, on October 26, 1942. He received extensive training and experience in image analysis while serving in the U.S. Navy from 1962 to 1971. He was assigned to the USS Manhattan during its test voyage through the Northwest Passage in 1969. Upon leaving the Navy he continued to pursue interests in sea-ice research at the U.S. Coast Guard Research and Development Center, and then joined the Naval Oceanographic and Atmospheric Research Laboratory's (NOARL) Polar Oceanography Branch, Hanover, NH, in 1978 , where he has been involved with Arctic remote sensing programs which utilize a variety of satellite, aircraft, and surface sensors. His current research applies advanced processing techniques to active and passive microwave imagery of sea ice to solve Navy-related problems. 